\title{
Estudo das relações anatomocirúrgicas entre os nervos laríngeos recorrentes e o ligamento de Berry
}

\author{
Anatomy surgical study of relations between the recurrent laryngeal nerves and \\ the ligament of Berry
}

João Bosco Botelho, TCBC-AM; Danilo Monteiro Vieira²; Diego Monteiro de Carvalho3; Marcus Brunner Pereira Batista²

R E S U M O

\begin{abstract}
Objetivo: Estudar as relações anatômicas do nervo laríngeo recorrente (NLR) com o ligamento de Berry (LB), e assinalar as diferentes formas de apresentação das relações entre essas duas estruturas. Métodos: Estudo realizado a partir da dissecção cervical anterior de 22 cadáveres, nos anos de 2009 e 2010, com estabelecimento das frequências de apresentações anatômicas das relações entre o NLR e o ligamento de Berry, na seguinte categorização: Tipo I ou intraligamentar: o nervo e/ou seus ramos eram visualizados na substância conjuntiva do ligamento; Tipo IIA ou lateral: nervo e/ou seus ramos apresentavam-se laterais ao ligamento; Tipo IIB ou lateral justaligamentar: nervo e/ou seus ramos estavam laterais e em contato com o ligamento, sem penetrá-lo; Tipo III ou medial: nervo e/ou seus ramos eram visualizados após a dissecção completa do ligamento em direção látero-medial. Resultados: 0 estudo analisou 41 NLR, sendo: oito (19,5\%) do Tipo l; 20 (48,8\%) do Tipo IIA; cinco (12,2\%) Tipo IIB e oito (19,5\%) do Tipo III. Dos 19 (86,3\%) NLR dissecados bilateralmente no mesmo cadáver, 11 (57,8\%) eram discordantes em relação ao outro lado do pescoço quanto à classificação. Conclusão: Pela sua proximidade com o NLR e pelas variáveis anatômicas dessa topografia, o ligamento de Berry não deve ser considerado um parâmetro seguro para reparo e preservação do nervo laríngeo recorrente, não sendo recomendada a dissecção indiscriminada látero-medial do ligamento, sem a visualização direta do nervo.
\end{abstract}

Descritores: Glândula tireoide. Tireoidectomia. Anatomia. Nervo laríngeo recorrente. Ligamentos.

\section{INTRODUÇÃO}

A s doenças cirúrgicas da glândula tireoide, em especial os bócios de grande volume, representam importantes problemas de saúde pública, principalmente em áreas geográficas bociogênicas ${ }^{1}$. Como consequência do tamanho dos bócios, alguns com duzentos gramas, o tratamento cirúrgico inclui possibilidades técnicas de acidentes transoperatórios e complicações pós-operatórias ${ }^{2,3}$.

O nervo laríngeo recorrente (NLR) origina-se do nervo vago, décimo par craniano, em níveis diferentes nos dois lados do pescoço, salvo na má formação congênita, quando o nervo laríngeo não é recorrente, isto é, após a saída do nervo vago, penetra na laringe sem recorrer no tórax ${ }^{4}$. Os NLR direito e esquerdo ascendem no espaço anatômico entre a traqueia e o esôfago, dispondo-se medial ao lobo correspondente da glândula tireoide ${ }^{5-7}$.

Os NLR dividem-se em dois ou mais ramos e passam junto à borda inferior do músculo constritor inferior da faringe, próximo à artéria laríngea inferior, penetrando a laringe atrás da membrana cricotireoidea ${ }^{6,7}$.
Relaciona-se topograficamente, de forma variável, com a artéria tireóidea inferior e com a glândula tireoide, e, mesmo que provavelmente nunca esteja envolvida na sua substância glandular normal, tal proximidade imprime maior chance de lesão nervosa em tireoidectomias totais e parciais ${ }^{5-7}$. Do mesmo modo, os NLR mantêm íntima relação com o ligamento de Berry: o espessamento da fáscia pré-traqueal que liga os lobos tireoideanos à traqueia e à parte inferior da cartilagem cricoide. Os NLR somente foram identificados laterais ao ligamento de Berry e eventualmente intraligamentares ${ }^{5-8}$.

A secção unilateral ou bilateral do NLR como possibilidade de acidente transoperatório nos bócios de grande volume, resulta na paralisia dos músculos intrínsecos da laringe, com exceção do cricotireoideo, determinando alterações na qualidade da voz e transtornos respiratórios ${ }^{5-7}$

O presente trabalho objetivou estudar as relações anatômicas do nervo laríngeo recorrente com o ligamento de Berry, e assinalar as diferentes formas de apresentação das relações entre essas duas estruturas. 


\section{MÉTODOS}

Trata-se de um estudo anatômico, topográfico e descritivo, realizado durante os anos de 2009 e 2010, por meio de dissecção da região cervical de 22 cadáveres frescos, não formolizados. Aprovado pelo Comitê de Ética em Pesquisa da Escola Superior de Ciências da Saúde da Universidade do Estado do Amazonas/UEA, protocolo n 45/ 2009.

As dissecções expuseram as regiões anterolaterais direitas e/ou esquerdas do pescoço, com o objetivo de visualizar a relação entre o ligamento de Berry (LB) e o nervo laríngeo recorrente (NLR).

Aproveitou-se a incisão mediana xifopúbica, utilizada nas necropsias, ampliando-a superiormente até a região supra-hioidea, seguida de: 1) dissecção dos músculos infra-hiodeos, miotomia transversal das porções craniais e exposição da glândula tireoide em sua totalidade; 2) identificação e dissecção do nervo laríngeo recorrente desde o quinto ou sexto anel traqueal na proximidade da goteira tráqueo-esofágica até sua penetração na laringe; 3) identificação e secção dos pedículos tireoidianos superiores e inferiores para observação da relação do nervo com o LB; 4) tração e dissecção da região látero-posterior do lóbulo da glândula até identificação do LB; 5) inclusão de uma fita métrica no campo quando os nervos eram laterais ao ligamento; 6) registro fotográfico da relação do nervo com o ligamento; 7) síntese da pele.

As diferentes relações anatômicas entre o nervo e o ligamento foram assim categorizadas: Tipo I ou intraligamentar: nervo e/ou seus ramos eram visualizados na substância conjuntiva do ligamento; Tipo IIA ou lateral: nervo e/ou seus ramos apresentavam-se em topografia lateral ao ligamento; Tipo IIB ou lateral justaligamentar: nervo e/ou seus ramos eram visualizados laterais e em contato com o ligamento, sem penetrá-lo; Tipo III ou medial: nervo e/ou seus ramos eram visualizados após a dissecção completa do ligamento em direção láteromedial.

Os critérios de inclusão foram cadáveres frescos, não formolizados, de qualquer sexo e idade, que, quando dissecados, demonstraram, de forma clara, a relação entre o nervo laríngeo recorrente e o ligamento de Berry.

Cadáveres que possuíam dismorfismo da glândula tireoide e traumas cervicais que impossibilitassem a observação da relação do ligamento de Berry com o nervo laríngeo recorrente, foram excluídos do estudo.

\section{RESULTADOS}

Foram estudadas as relações anatômicas de 44 NLR (Figuras 1,2,3). Três amostras (6,8\%) do ramo laríngeo direito foram excluídas do estudo.

Dos cadáveres analisados, 20 (90,9\%) eram do sexo masculino e dois $(9,1 \%)$ do feminino. A variável ida-

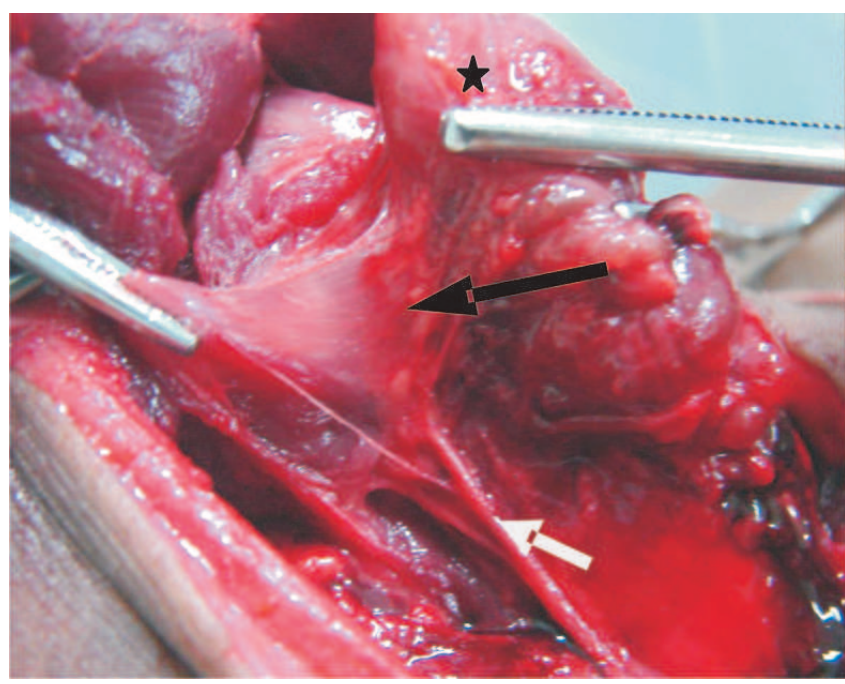

Figura 1 - NLR e ligamento de Berry tipo / - intraligamentar. Glândula tireoide (estrela); ligamento de Berry (seta preta); nervo laríngeo recorrente (seta branca).

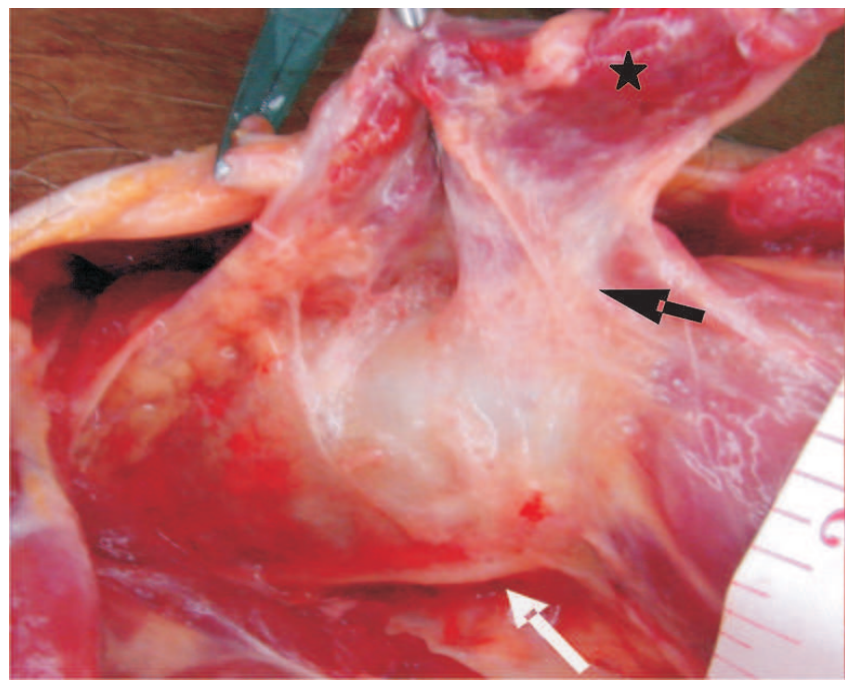

Figura 2 - NLR e ligamento de Berry tipo IIA - Lateral. Glândula tireoide (estrela); ligamento de Berry (seta preta), nervo laríngeo recorrente (seta branca).

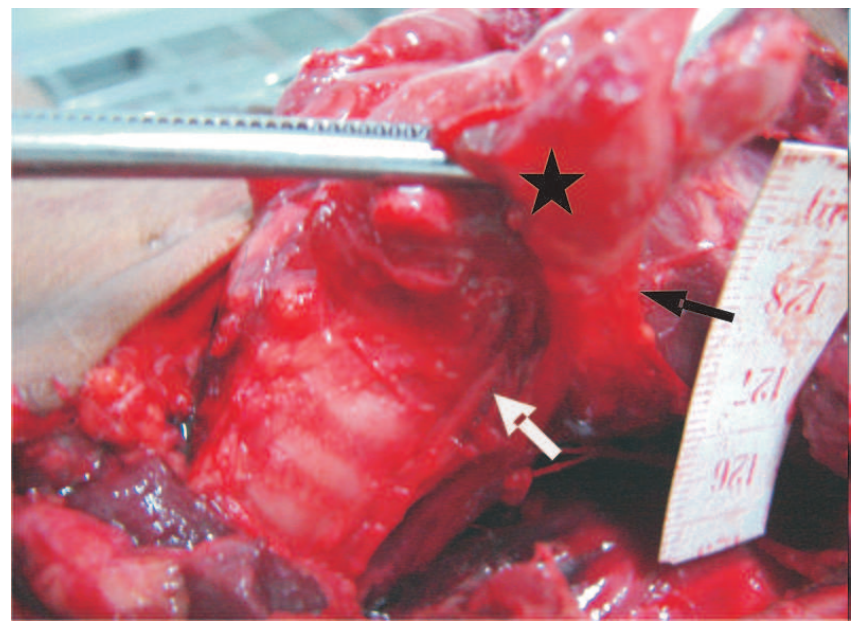

Figura 3 - NLR e ligamento de Berry tipo III. Glândula tireoide (estrela), - ligamento de Berry (seta preta), nervo laríngeo recorrente (seta branca). 
de foi analisada pela estimativa do perito, já que todos eram indigentes, com a maioria dos cadáveres sendo classificados como adultos jovens (H" $14-55$ anos).

Foram analisadas, bilateralmente, 19 (86,3\%) regiões cervicais e $11(57,8 \%)$ delas eram discordantes quanto à classificação do NLR em relação ao outro lado do pescoço. A frequência de apresentação das relações entre o NLR e o ligamento de Berry pode ser vista na tabela 1.

Nos NLR classificados no subtipo IIA (20/48,8\%), procedeu-se com a medida das distâncias entre o nervo e o LB, conforme demonstra a tabela 2 .

\section{DISCUSSÃO}

Os nervos laríngeos recorrentes, pela relevância funcional, são de identificação obrigatória durante a realização das tireoidectomias parciais e totais ${ }^{1-4,9-14}$.

Os bócios de grande volume, notadamente nos pacientes residentes no Amazonas, impõem outras formas de relação anatômica entre o nervo e o ligamento de Berry, justificam maior atenção do cirurgião ${ }^{1-4,15}$.

Sasou et al. ${ }^{10}$, em 486 tireoidectomias e 25 necropsias, e Cakir et al. ${ }^{13}$, em 65 autópsias, encontraram todos os nervos laterais ao ligamento de Berry e nenhum intraligamentar, entretanto a posição justaligamentar do nervo laríngeo recorrente também já foi descrita 8,9 . Leow e Webb ${ }^{8}$ e Lekacos et al. ${ }^{9}$ descreveram a maioria dos NLR como muito próximos ao ligamento de Berry, com as mai- ores distâncias variando de 0 a $0,5 \mathrm{~cm}$, sem o tipo intraligamentar.

Constatamos que 25 (61\%) NLR eram laterais ao ligamento (Figura 2), e desses, cinco (12,2\%) eram do tipo IIB ou justaligamentar, estes achados são semelhantes aos de Wafae et al. ${ }^{11}$ De forma concordante com a literatura ${ }^{11,16,17}$, oito (19,5\%) NLR eram intraligamentar ou tipo I (Figura 1). Berlin et al. ${ }^{17}$, em $25 \%$, Wafae et al. ${ }^{11}$, em $10 \%$, e Yalçin et al. ${ }^{16}$, em 2\% das amostras, identificaram esses mesmos tipos nervosos. Ao contrário de Leow e Webb ${ }^{8} \mathrm{e}$ Lekacos et al. ${ }^{9}$, registramos distâncias até $1,6 \mathrm{~cm}$ entre 0 nervo laríngeo recorrente e o ligamento de Berry.

A nova proposta classificatória desse estudo inclui uma terceira relação entre NLR e o ligamento de Berry não mencionada por outros autores: o nervo laríngeo recorrente em posição medial, identificada em oito $(19,5 \%)$ dos nervos dissecados (Figura 3).

Essa divergência de resultados pode ser explicada pelos imprecisos limites do ligamento, ou ainda, pelas diferentes técnicas de dissecção em campo operatório ou em peças anatômicas.

Esse trabalho reforça a necessidade de que a dissecção látero-medial do ligamento de Berry deve ocorrer, obrigatoriamente, após a clara identificação do NLR, já que o segmento cranial no nervo pode estar medial ao ligamento. Como consequência, esse estudo anatômico, também pode auxiliar os jovens cirurgiões na compreensão das relações anatômicas entre o ligamento de Berry e os nervos laríngeos, especialmente, na dissecção láteromedial do ligamento.

Tabela 1 - Frequência de apresentação das diferentes relações entre o NLR e o ligamento de Berry ( $n=41)$.

\begin{tabular}{lcrrrrc}
\hline Tipo* & Esquerdo & $\%$ & Direito & $\%$ & Total & $\%$ \\
\hline I & 4 & 9,8 & 4 & 9,8 & 8 & 19,5 \\
IIA & 10 & 24,4 & 10 & 24,4 & 20 & 48,8 \\
IIB & 4 & 9,8 & 1 & 2,4 & 5 & 12,2 \\
III & 4 & 9,8 & 4 & 9,8 & 8 & 19,5 \\
Total & 22 & 53,8 & 19 & 46,4 & 41 & 100 \\
\hline
\end{tabular}

* Tipo I ou intraligamentar; Tipo IIA ou lateral; Tipo IIB ou lateral justaligamentar; Tipo III ou medial.

Tabela 2 - Medidas das distâncias entre o NLR e o ligamento de Berry dos nervos Tipo IIA ( $\mathrm{n=20}$.

\begin{tabular}{lccccc}
\hline Variável & $\mathbf{N}$ & Média $(\mathrm{cm})$ & Mediana $(\mathrm{cm})$ & Máximo $(\mathrm{cm})$ & Mínimo $(\mathrm{cm})$ \\
\hline DNLR-LBE* & 10 & 0,69 & 0,8 & 1,6 & 0,2 \\
DNLR-LBD** & 10 & 0,59 & 0,8 & 1,3 & 0,3 \\
\hline
\end{tabular}

*DNLR-LBE - Distância entre o nervo laríngeo recorrente e o ligamento de Berry do lado esquerdo.

**DNLR-LBD - Distância entre o nervo laríngeo recorrente e o ligamento de Berry do lado direito. 


\begin{abstract}
Objective: To study the anatomical relations of the recurrent laryngeal nerve (RLN) with the ligament of Berry (LB), and point out the different ways of presenting the relationship between these two structures. Methods: We conducted a study with anterior cervical dissection of 22 corpses, in the years 2009 and 2010, with attention towards the frequency of presentation of anatomical relations between the NLR and Berry ligament, with the following classification: Type I or intraligamentary: the nerve and / or its branches were visualized in the ligament substance conjunctiva; Type IIA or lateral: nerve and / or its branches lateral to the ligament; Type IIB or lateral paraligamentary: nerve and / or its side branches and in contact with the ligament without penetrating it; Type III or medial: nerve and / or its branches visualized after complete dissection of the ligament, in lateral-medial position. Results: The study analyzed 41 RLNs, which comprised eight (19.5\%) of type I, 20 (48.8\%) Type II, five (12.2\%) Type IIB and eight (19.5\%) type III. Of the 19 (86.3\%) RLNs dissected bilaterally in the same cadaver, 11 (57.8\%) displayed a classification in one side of the neck different from the one of the other side. Conclusion: Due to its proximity to the RLN and the anatomical variability, the Berry ligament should not be considered a reliable parameter for repair and preservation of the recurrent laryngeal nerve. Hence, the indiscriminate latero-medial dissection of the ligament is not recommended without direct visualization of the nerve.
\end{abstract}

Key words: Thyroid gland. Thyroidectomy. Anatomy. Recurrent laryngeal nerve. Ligaments.

\section{REFERENCIAS}

1. Botelho JB. Pathologie de la glande thyroide. In: Botelho JB, Gehanno P, editors. Otorhinolaryngologie et chirurgie cervicofaciale à I'usage dês étudiants. Paris: Edk; 2002. p. 61-90.

2. Botelho JB, Cançado AR, Sousa EA. Características macroscópicas, localização e suprimento vascular das glândulas paratireoides cervicais. Prêmio Nacional de Anatomia Humana 2002 Prof. Garcia do Prado. Rio Grande do Sul: Pontifícia Universidade Católica; 2002.

3. Botelho JB. Patologia da glândula tireoide. In: Botelho JB. Otorrinolaringologia e cirurgia de cabeça e pescoço para estudantes. Manaus: Universidade Federal do Amazonas; 2000. p. 325-98.

4. Botelho JB, Anjos GS, Pires GP, Ferreira DMR, Leite TO, Resende GAS. Protocolo anatômico-cirúrgico das relações entre os nervos laríngeos recorrentes e as artérias tireoideas inferiores: estudo em 79 tireoidectomias. Rev Col Bras Cir. 2007;34(3):142-6.

5. Gardner E, Gray DJ, O'Rahilly R. Anatomia. Estudo regional do corpo humano. $4^{a}$ ed. Rio de Janeiro: Guanabara Koogan; 1988.

6. Rouvière HD. Anatomie humaine descriptive, topographique et fonctionnelle. Tête et cou. Paris: Masson; 1984.

7. Testut L, Latarjet A. Tratado de anatomia humana. Barcelona: Salvat; 1959.

8. Leow CK, Webb AJ. The lateral thyroid ligament of Berry. Int Surg 1998;83(1):75-8.

9. Lekacos NL, Tzardis PJ, Sfikakis PG, Patoulis SD, Restos SD. Course of the recurrent laryngeal nerve relative to the inferior thyroid artery and the suspensory ligament of Berry. Int Surg. 1992;77(4):287-8.

10. Sasou S, Nakamura S, Kurihara H. Suspensory ligament of Berry: its relationship to recurrent laryngeal nerve and anatomic examination of 24 autopsies. Head Neck. 1998;20(8):695-8.

11. Wafae N, Ruiz CR, Wafae GC, Vorobieff A. Estudo anatômico de pontos críticos no trajeto do nervo laríngeo recorrente: ligamento suspensor da glândula tireoide (ligamento de Berry) e cápsula da glândula tireoide. Mundo saúde. 2009;33(1):26-30.
12. Monfared A, Gorti G, Kim D. Microsurgical anatomy of the laryngeal nerves as related to thyroid surgery. Laryngoscope. 2002;112(2):386-92.

13. Cakir BO, Ercan I, Sam B, Turgut S. Reliable surgical landmarks for the identication of the recurrent laryngeal nerve. Otolaryngol Head Neck Surg. 2006;135(2):299-302.

14. Bergamaschi R, Becouarn G, Ronceray J, Arnaud JP. Morbidity of thyroid surgery. Am J Surg. 1998;176(1):71-5

15. Botelho JB, Cattebeki L, Cattebeki T, Anjos GS; Lima RM, Barbosa MF, et al. Histopathologie de 573 thyroïdectomies réalisées entre 1976 et 1998 Manaus - Brésil. Revue Officielle de La Societé Française D'ORL et de Chirurgie de La Face et Cou. 2001; 66(2):47-9.

16. Yalçin B, Tu!cu H, Cantürk N, Kurt B, Ozan H. Critical course of the anterior laryngeal branch of the inferior laryngeal nerve. ANZ J Surg. 2006;76(6):481-3.

17. Berlin DD. The recurrent laryngeal nerves in total ablation of the normal thyroid gland - an anatomical and surgical study. Surg Gynecol Obstet. 1935;60:19-26.

Recebido em 27/02/2012

Aceito para publicação em 15/04/2012

Conflito de interesse: nenhum

Fonte de financiamento: Fundação de Amparo à Pesquisa do Estado do Amazonas (FAPEAM)

\section{Como citar este artigo}

Botelho JB, Vieira DM, Carvalho DM, Batista MBP. Estudo das relações anatomocirúrgicas entre os nervos laríngeos e o ligamento de Berry. Rev Col Bras Cir. [periódico na Internet] 2012; 39(5). Disponível em URL: http://www.scielo.br/rcbc

\section{Endereço para correspondência:}

João Bosco Lopes Botelho

E-mail: joaoboscobotelho@gmail.com 